\title{
CHATBOTS: A EFETIVIDADE DA INTELIGÊNCIA ARTIFICIAL NO APOIO À SAÚDE MENTAL
}

\author{
Fábio Meurer'1, Patrícia Bergamasco², Lucas França Garcia ${ }^{3}$ \\ 'Acadêmico do Curso de Mestrado em Promoção da Saúde do Centro Universitário de Maringá - UNICESUMAR. \\ fabio.meurer@unicesumar.edu.br \\ ${ }^{2}$ Acadêmica do Curso de Mestrado em Promoção da Saúde do Centro Universitário de Maringá - UNICESUMAR. \\ drapatricia22@hotmail.com \\ 3Orientador, Doutor em Medicina - UFRGS. Pós-Doutorado em Promoção da Saúde - UNICESUMAR \\ lucas.garcia@unicesumar.edu.br
}

\section{RESUMO}

O acesso a serviços e ao tratamento da saúde mental continua sendo um problema global. Os chatbots, também conhecidos como agentes de conversação ou assistentes digitais, são programas de software baseados em inteligência artificial projetados para interagir com pessoas de maneira a simular um diálogo. Há várias formas de interação, como aplicativos de texto, áudio e vídeo. Neste artigo, discutimos as publicações, funcionalidades disponíveis e a efetividade das plataformas digitais interativas voltadas ao cuidado da saúde mental. Este é um estudo de revisão desenvolvido em ambiente online. Foi realizada a busca de artigos nas bases de dados Pubmed e Scielo com os termos "chatbot" e "mental health" para a definição de publicações relacionadas ao tema dos últimos 3 anos. Foram extraídos os resultados referentes a efetividade dos programas interativos de conversação no manejo do tratamento dos transtornos mentais. Os chatbots automatizados representam um grande potencial ao fornecer ajuda útil nas dificuldades de saúde mental. A grande maioria dos estudos demonstrou resultados favoráveis, embora quase a totalidade deles fez ressalvas quanto a necessidade de maiores pesquisas para a validação dos protocolos.

PALAVRAS-CHAVE: Comunicação; Agente interativo; Bem-estar; Assistente digital.

\section{INTRODUÇÃO}

Um relatório da Organização Mundial da Saúde mostra que a depressão acomete quase $5 \%$ da população global e está associada à redução da qualidade de vida. Os longos períodos de espera, a estigmatização e a falta de recursos são apontados como objeções e obstáculos a serem transpostos. Diante disso, considerando-se que os transtornos mentais são um problema crescente de saúde pública, surgem iniciativas para o seu enfrentamento. Todavia, não há como disponibilizar a terapia presencial nos moldes tradicionais e com a agilidade necessária. A falta de profissionais capacitados é um dos maiores impeditivos. $\mathrm{O}$ acesso a serviços e ao tratamento da saúde mental continua sendo um desafio e a depressão permanece como uma das principais causas de afastamento laboral por incapacidade (CHAIX et al., 2019) (VAIDYAM et al., 2019). Na tentativa de desenvolver soluções, nos últimos 20 anos houve um significativo aumento na divulgação de tecnologias voltadas ao cuidado emocional. Mais recentemente, foram disponibilizados vários agentes de conversação, ou chatbots, totalmente automatizados. São plataformas de texto, facilmente acessadas por meio de um aplicativo ou do Facebook (INKSTER; SARDA; SUBRAMANIAN, 2018).

Chatbots, também conhecidos como agentes de conversação ou assistentes digitais, são programas de software baseados em inteligência artificial projetados para interagir com pessoas de maneira a simular uma conversa. Eles são frequentemente usados na triagem de atendimento ao cliente, mas na área da saúde, podem trocar informações com os pacientes, funcionando como um acompanhante, ou exercer funções de triagem, coleta de 
dados e adesão ao tratamento. Existem diversas formas de interação, como serviços de texto (mensagens e salas de bate-papo) ou serviços de áudio, como Siri, Alexa, Cortana e Google Assistant (BIBAULT et al., 2019). Os programas são empáticos e desenhados de forma que aumentam a aderência na medida em que se envolvem com os usuários parecendo interações da vida real (KRETZSCHMAR et al., 2019). Por outro lado, eles precisam preservar a privacidade dos usuários, promover resultados positivos e ainda atentar para o fato de que há uma tendência natural de substituir o trabalho do profissional especializado. É necessário que haja mecanismos de alerta, principalmente na prevalência de problemas psiquiátricos mais graves (INKSTER; SARDA; SUBRAMANIAN, 2018) (PALANICA et al., 2019).

\section{METODOLOGIA}

Este é um estudo de revisão desenvolvido em ambiente online. Foi realizada a busca de artigos nas bases de dados Pubmed e Scielo com os termos "chatbot" e "mental health" para a definição de publicações relacionadas ao tema dos últimos 3 anos. Foram consideradas pertinentes 10 publicações randomizadas. Após realizada a revisão bibliográfica foram extraídos os resultados referentes a efetividade dos programas interativos de conversação no manejo do tratamento dos transtornos mentais. Os resultados foram descritos conforme a conclusão apresentada pelos autores.

\section{RESULTADOS E DISCUSSÃO}

Morris et al. (2018) desenvolveram um agente de conversação que pudesse expressar empatia de maneira a simular capacidades humanas. O sistema reproduziu uma abordagem de empatia por meio de respostas pré-existentes ao usuário. Foram coletados dados de 37.169 usuários. Foi realizado também um experimento para testar se a percepção do usuário mudaria conforme houvesse interação humana ou eletrônica. A maior parte das interações foram consideradas aceitáveis, no entanto, os usuários preferiram as interações humanas. O sistema ilustra uma maneira das máquinas construírem enunciados empáticos e personalizados, porém, o design apresentou limitações significativas e mais pesquisas serão necessárias. O estudo sugere que, mesmo em condições ideais, os agentes não humanos podem ter dificuldades em expressar empatia. Há ainda implicações éticas, bem como seus potenciais efeitos iatrogênicos. O sistema foi considerado efetivo, mas ainda necessitando de melhorias.

Fitzpatrick, Darcy e Vierhile (2017) em seu estudo relataram que os aplicativos de terapia cognitivo-comportamental demonstraram eficácia, mas apresentaram uma baixa adesão. Os agentes de conversação podem mostrar-se convenientes ao oferecer ajuda a qualquer hora. O objetivo do estudo foi determinar a viabilidade, a aceitabilidade e a eficácia de um agente totalmente automatizado, voltado a estudantes universitários que se identificam com sintomas de ansiedade e depressão. Foram recrutados 70 universitários, entre 18 e 28 anos, divididos em dois grupos randomicamente: o primeiro iria interagir com um agente de conversação baseado em texto, com conteúdos de autoajuda derivados da terapia cognitivo-comportamental. O segundo grupo seria direcionado ao e-book do Instituto Nacional de Saúde Mental, "Depressão em Estudantes Universitários", como um grupo controle, apenas informativo. A análise dos resultados revelou uma diferença significativa no primeiro grupo, o qual reduziu os sintomas de depressão. Os comentários dos usuários sugerem que o programa interativo foi mais influente em sua aceitabilidade do que a terapia tradicional em si, ou seja, parece ser uma maneira envolvente e eficaz de fornecer suporte terapêutico. 
Ho, Hancock e Miner (2018) neste estudo informaram os participantes de que teriam uma conversa com um chatbot ou com uma pessoa. Empregaram um método de interação no qual informava-se que o parceiro seria um computador, quando na realidade uma pessoa escondida é que interagiria com os participantes. Em outras palavras, alguns participantes foram informados de que teriam uma conversa com um chatbot e alguns foram informados de que teriam uma conversa com uma pessoa, mas em todos os casos o interlocutor era uma pessoa. Essa estratégia objetivava reduzir limitações e percepções de identidade. Houve também duas abordagens de interação, uma emocional e outra objetiva. Os resultados demonstraram que nas situações emotivas os participantes revelaram mais suas emoções e compartilharam informações mais pessoais independentemente do agente interativo. Com relação ao interlocutor, eles usaram uma linguagem mais clara e simples nas interações com os supostos chatbots, usaram menos conjugações e menor fluência verbal. Porém, em todas as variáveis estudadas os resultados foram equivalentes, ou seja, os participantes que interagiram com os "chatbots" experimentaram benefícios emocionais, relacionais e psicológicos tanto quanto os participantes que interagiram com um parceiro humano.

Inkster, Sarda e Subramanian (2018) neste estudo tiveram como objetivo apresentar uma avaliação preliminar da eficácia e do engajamento relativos a um aplicativo de conversação, baseado em texto, com enfoque no bem-estar mental. Os usuários com sintomas de depressão instalaram voluntariamente o aplicativo. A análise quantitativa mediu seu impacto comparando a melhora nos sintomas de depressão entre usuários graves e leves. A análise qualitativa mediu o engajamento e a experiência do usuário. Avaliou também o desempenho de aprendizagem de máquina para detectar objeções do usuário durante as conversas. Os resultados demonstraram uma melhora significativamente maior nos casos graves em comparação aos casos leves. Além disso, mais de dois terços das respostas de feedback fornecidas consideraram a experiência útil e encorajadora. A eficácia e os níveis de envolvimento do aplicativo são promissores. No entanto, é necessário validar essas descobertas iniciais em amostras maiores e em períodos mais longos.

Kretzschmar et al. (2019) afirmam que chatbots estão se tornando mais acessíveis a qualquer pessoa com um smartphone. Os aplicativos e plataformas digitais são muito menos estigmatizantes em comparação aos serviços comuns de saúde mental, portanto eles podem representar um primeiro passo importante na busca por ajuda. Os autores sugerem os padrões mínimos a serem seguidos pelos programas de interação: devem garantir a segurança, respeitar a privacidade dos usuários e seguir protocolos baseados em evidências. Encorajam os desenvolvedores a usar as recomendações em conformidade com os padrões locais. O número crescente de chatbots sinaliza que existe uma demanda por apoio à saúde mental que não está sendo atendida pelos serviços tradicionais. As pessoas poderão depender cada vez mais de recursos digitais, o que reforça a importância de criar plataformas eficazes e eticamente responsáveis.

Vaidyam et al. (2019) realizaram uma revisão sistemática com o objetivo de explorar as evidências de agentes de conversação no campo da psiquiatria e seu papel na triagem, diagnóstico e tratamento das doenças mentais. Das bases de dados selecionadas, 1466 registros foram recuperados e 8 estudos preencheram os critérios de inclusão. Dois estudos adicionais foram incluídos na triagem da lista de referência num total de 10. De forma geral, o potencial dos agentes de conversação em uso psiquiátrico foi descrito como alto em todos os estudos. Os agentes de conversação mostraram-se particularmente interessantes em benefício da educação emocional. Além disso, o índice de satisfação foi alto em todos os estudos, sugerindo que eles seriam uma ferramenta eficaz e agradável no tratamento psiquiátrico. Os autores concluem que as evidências preliminares são favoráveis. Embora 
tenha sido evidenciada uma heterogeneidade nos estudos revisados, portanto pesquisas adicionais são necessárias.

\section{4}

\section{CONSIDERAÇÕES FINAIS}

Apesar das limitações mencionadas acima, os chatbots automatizados representam um grande potencial ao fornecer ajuda útil nas dificuldades de saúde mental. A grande maioria dos estudos demonstrou resultados favoráveis, embora quase a totalidade deles fez ressalvas quanto a necessidade de maiores pesquisas para a validação dos protocolos. Existem ainda, várias questões éticas associadas ao seu potencial. Há preocupações relacionadas a quem teria acesso a informações pessoais e conversas dos usuários; se o suporte digital é baseado em evidências; e com relação a segurança dos usuários em situações de emergência. Para que seu desenvolvimento não seja comprometido, os programas devem atender a um conjunto de padrões éticos mínimos em relação à privacidade, confidencialidade, eficácia e segurança.

\section{REFERÊNCIAS BIBLIOGRÁFICAS}

BIBAULT, J.-E.; CHAIX, B.; NECTOUX, P.; BROUARD, B. Healthcare ex Machina: Are conversational agents ready for prime time in oncology? Clinical and translational radiation oncology, v. 16, p. 55-59, maio 2019.

CHAIX, B.; BIBAULT, J.-E.; PIENKOWSKI, A.; DELAMON, G.; GUILLEMASSÉ, G.; NECTOUX, P.; BROUARD, B. When Chatbots Meet Patients: One-Year Prospective Study of Conversations Between Patients With Breast Cancer and a Chatbot. JMIR cancer, v. 5, n. 1, p. e12856, maio 2019.

FITZPATRICK, K. K.; DARCY, A.; VIERHILE, M. Delivering Cognitive Behavior Therapy to Young Adults With Symptoms of Depression and Anxiety Using a Fully Automated Conversational Agent (Woebot): A Randomized Controlled Trial. JMIR mental health, v. 4, n. 2, p. e19, jun. 2017.

HO, A.; HANCOCK, J.; MINER, A. S. Psychological, Relational, and Emotional Effects of Self-Disclosure After Conversations With a Chatbot. Journal of Communication, v. 68, n. 4, p. 712-733, 1 ago. 2018.

INKSTER, B.; SARDA, S.; SUBRAMANIAN, V. An Empathy-Driven, Conversational Artificial Intelligence Agent (Wysa) for Digital Mental Well-Being: Real-World Data Evaluation MixedMethods Study. JMIR mHealth and uHealth, v. 6, n. 11, p. e12106, nov. 2018.

KRETZSCHMAR, K.; TYROLL, H.; PAVARINI, G.; MANZINI, A.; SINGH, I. Can Your Phone Be Your Therapist? Young People's Ethical Perspectives on the Use of Fully Automated Conversational Agents (Chatbots) in Mental Health Support. Biomedical informatics insights, v. 11, p. 1178222619829083, 2019.

MORRIS, R. R.; KOUDDOUS, K; KSHIRSAGAR, R.; SCHUELLER, S. Towards an Artificially Empathic Conversational Agent for Mental Health Applications: System Design and User Perceptions. Journal of Medical Internet Research, v. 20, n. 6, p. e10148, 26 jun. 2018.

PALANICA, A.; FLASCHNER, P.; THOMMANDRAM, A.; LI, M.; FOSSAT, Y. Physicians' Perceptions of Chatbots in Health Care: Cross-Sectional Web-Based Survey. Journal of medical Internet research, v. 21, n. 4, p. e12887, abr. 2019. 
VAIDYAM, A. N.; WISNIEWISKI, H.; HALAMKA, J. D.; KASHAVAN, M.; TOROUS, J. B. Chatbots and Conversational Agents in Mental Health: A Review of the Psychiatric Landscape. Canadian journal of psychiatry. Revue canadienne de psychiatrie, $p$. 706743719828977, mar. 2019. 